\title{
Algorithm for Registering Point Cloud Data with Quadratic Surfaces
}

\author{
Nie Jianhui ${ }^{1}$ \\ ${ }^{1}$ School of Automation, Nanjing University of Posts and Telecommunications, \\ Nanjing 210023 \\ njh19@163.com
}

\begin{abstract}
Data registration is a common operation in object digitalization. This paper proposes an algorithm for registering point cloud data with such quadratic surfaces as planes and cylinders. In the proposed algorithm, the variation of neighboring points' normal vectors are utilized to detect data that is located at the intersection of different quadratic surfaces, which is then deleted from the point clouds. Next, region segmentation is done on remaining point clouds through the growth algorithm, and feature descriptors are created for each detected quadratic surface. Finally, rough registration of data is achieved via matching of feature descriptors, and then fine registration is achieved by optimizing rough registration results through the ICP algorithm. Experimental results show that the algorithm is insensitive to outliers, has low requirements on the original poses of data and that its registration success rate is higher than that of the original ICP algorithm.
\end{abstract}

Keywords: Point clouds; Quadratic; Registration; Segmentation

\section{Introduction}

Recent years of constant progress in structured light measuring technology lead to great need for multi-view data registration. Data registration refers to the process of finding the coordinate of the same point in the space using 3-D data collected from different views, and then restoring coordinate conversion between views. This process can be divided into two steps: rough registration and fine registration.

Rough registration: Creating feature descriptors is a popular method for rough registration of data. This type of method first recognizes the feature points from the entirety of point clouds using certain metrics. Next, the neighbors of feature points are utilized to create a globally unique feature descriptor. Finally, rough registration is achieved by matching feature descriptors of the two point clouds. Sipiran et. al., [1] performed feature detection by extending the concept of angular point in the image to the 3-D space. Mokhtarian et. al., [2] detected feature points by finding the one-loop neighbor with maximal curvature in the mesh surface. Gal et. al., [3] defined the linear combination of the sum and variances of neighboring point's curvature as the criterion of feature point detection. Zaharescu et. al., [4] and J. Stuckler et. al., [5] achieved detection of feature points in the non-uniform mesh surface by performing multi-scale operations on the curvature and the normal vector, respectively. For creation and matching of feature descriptor, Tombari et. al., [6] associated each feature descriptor with a local coordinate system based on the direction of maximum principal curvature, and performed 3-D histogram statistics. Sukno et. al., [7] proposed a method which created feature descriptors via asymmetric statistics, and the descriptors from their method was rotational invariant. Mian et. al., [8] devised a feature descriptor that can remain stable in the case of isometric deformation. Through statistical analysis of surface message in the cubic grid, 
their method produced better matching results than the Spin images. For more details on feature point extraction and feature descriptor creation, please refer to [9].

Another type of methods for rough registration of data relies on the RANSAC-based algorithms. Feasibility of these algorithms have been proved in [10-11]. But the time complexity of them is $\mathrm{O}(\mathrm{N} 3)$ and is inefficient in processing point clouds with large overlaps. Thus, many algorithms were proposed to reduce the time complexity of these methods, such as the branch-and-boundary algorithm [12], the normal vector space layered representation algorithm [13], the random non-linear optimization algorithm [14], and the 4PCS algorithm [15]. These algorithms managed to reduce the complexity to $\mathrm{O}(\mathrm{N} 2)$. The advent of Super 4PCS [16] achieved linear complexity.

Although many works have been done on rough registration of data, how to match stably and stably in the presence of noise and outlier remains an issue that needs to be solved.

Fine registration: fine registration is done to further optimize results of rough registration. Fine registration algorithms are more mature than rough registration algorithms. The most common algorithm for fine registration is the iterative closest points algorithm (ICP) [17-18]. By defining closest points as the matching points, ICP iteratively computes the rigid transformation relationship between two views. Major improvements on ICP include the search scheme for closest points, deletion of incorrectly matched points, and the decision on convergence condition. Latest improvements are as follows. [19] improved the algorithm's termination condition by analyzing local minimum obtained through multiple ICP iterations. A sparse algorithm was proposed in [20] to enhance adaptability of ICP to outliers. [21] increased the algorithm's operation speed through parallel speedup.

Quadric surfaces (e.g., plane, sphere, cylinder, circular cone) usually occur in the actually scanned point clouds and especially in the point clouds with buildings, CAD work-pieces and other artificial objects. To the best of our knowledge, there is no literature investigating potentials of these quadratic surfaces for facilitating data registration. This paper proposes an algorithm for registering point clouds containing quadratic surfaces. The proposed algorithm begins with detecting quadratic surfaces in point clouds through region segmentation. Next, the proposed algorithm creates and matches feature descriptors to match quadratic surfaces, achieving registration of two point clouds. In the proposed algorithm, creation of feature descriptors is based on quadratic surface fitting. So, it outperforms the traditional methods in terms of original registration accuracy and tolerance to noise.

\section{Algorithm Steps}

Figure 1, shows steps of the proposed algorithm. Given two point clouds with quadratic surfaces, the algorithm first performs region segmentation on point clouds. Next, the type of quadratic surfaces in each region is predicted through optimal fitting. Feature descriptors are created for each region. Finally, rough registration is done by matching feature descriptors of the two point clouds and fine registration is done by the ICP algorithm. 


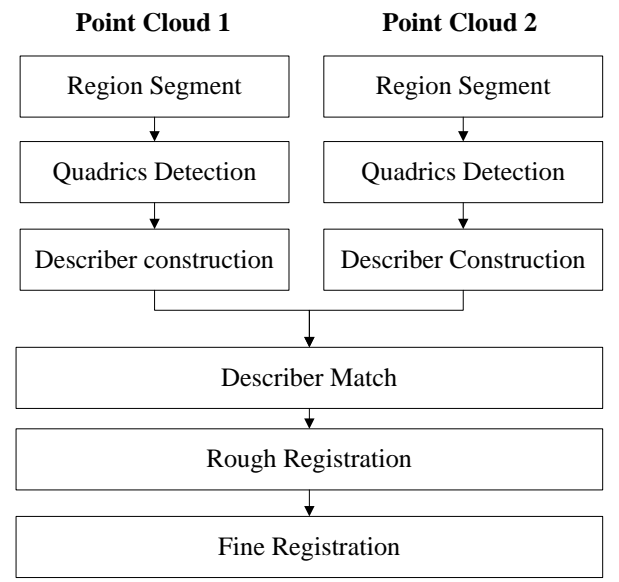

Figure 1. Algorithm Steps

\subsection{Region Segmentation}

The proposed method first removes the points near the intersection of different regions from the entirety of point clouds. Deliberate removal of these points causes a crack in point clouds. Then, region segmentation can be done using the growth algorithm.

The surfaces fluctuate greatly at the intersection of different regions. A metric for measuring fluctuation of surfaces is needed to recognize these points from the entirety of point clouds. Based on its definition, curvature is a direct representation of how greatly the surface fluctuates. Some data needs to be first parameterized to compute curvature of point clouds. The parametric surfaces should then be fitted (e.g., quadratic surface or spline surface). Finally, the curvature of the parametric surface at the corresponding point is defined as the calculation result. Despite its effectiveness for noise-free point clouds, the curvature method is prone to be affected by noise and outlier, due to the use of the first- and second-order differentials of parametric surfaces. Another method is to substitute surface variation for curvature. Although it has the ability to alleviate influence of noise by enlarging the computing neighborhood, its calculation result is not physically meaningful. Hence, this paper proposes to represent curvature of surface by calculating variation of normal vector within the neighborhood. On the one hand, calculation of normal vector is easy and accurate. On the other hand, the calculation result has clear physical meanings.

For any point $p$ in the point cloud $\mathbf{P}$ at an average sampling interval of $\delta$, the curvature of surface can be computed as follows: first, find the neighboring points Nbp within $10 \delta$ from $p$; then, sort out $\mathrm{Nbp}$ according to the angle of its normal vector to the normal vector of $p$; finally, we define the average of the first $2 \mathrm{n} / 3$ angles as the final result, where $n$ is the cardinal number of the set $\mathrm{Nbp}$.

After obtaining the fluctuate of surface, point clouds at the intersection of surfaces can be recognized through threshold and then deleted from the entirety of point clouds, generating point cloud $\mathrm{P}$ - without junction points. Then, region segmentation can be done using the distance-based regional growth algorithm: At first an empty growth list is generated, and an unprocessed point randomly selected from P- is push into the growth list. Then, take the first element of the growth list and set its status as "processed", and add its neighbors within $3 \delta$-radius into the growth list iteratively. Finally, growth of the current region ends when the growth list is empty. Repeat all above steps until all points are labeled "processed" and the region segmentation can be done. 


\subsection{Quadratic Surface Fitting}

The general form of any quadratic surface in the 3D space can be written as:

$$
\begin{aligned}
f(x, y, z)= & a_{11} x^{2}+a_{22} y^{2}+a_{33} z^{2}+2 a_{12} x y+2 a_{13} x z \\
& +2 a_{23} y z+a_{14} x+a_{24} y+a_{34} z+a_{44}=0
\end{aligned}
$$

Coefficient conversion can yield the objective equation in (2). Each coefficient can thus be computed through least squares.

$$
\sum_{i=1}^{n} e_{i}^{2}=\sum_{i=1}^{n}\left(\begin{array}{l}
a_{11}^{\prime} x_{i}^{2}+a_{22}^{\prime} y_{i}^{2}+a_{33}^{\prime} z_{i}^{2}+2 a_{12}^{\prime} x_{i} y_{i}+2 a_{13}^{\prime} x_{i} z_{i} \\
+2 a_{23}^{\prime} y_{i} z_{i}+a_{14}^{\prime} x_{i}+a_{24}^{\prime} y_{i}+a_{44}^{\prime}-\left(-z_{i}\right)
\end{array}\right)^{2}
$$

where $\mathrm{i}=1,2,3 \cdots \mathrm{n}$, and $\mathrm{n}$ denotes the number of points in the fitting region.

After the general equation is obtained, it needs to be converted into a standard equation using the quadratic form theory to determine the type of the quadratic surface. According to the report in [22], for quadratic surfaces that actually exist, when performing transformation on the original coordinate system $\mathrm{C}$ by choosing a proper orthogonal transform matrix $\mathrm{T}$, they can always be simplified into one of the eight forms in Table 1 .

Table 1. Standard Quadratic Surface Equations

\begin{tabular}{cccc}
\hline Types & Equations & Types & Equations \\
\hline Ellipsoid & $\frac{x^{2}}{a^{2}}+\frac{y^{2}}{b^{2}}+\frac{z^{2}}{c^{2}}=1$ & Elliptic paraboloid & $\frac{x^{2}}{a^{2}}+\frac{y^{2}}{b^{2}}-z=0$ \\
Hyperbolic & $\frac{x^{2}}{a^{2}}-\frac{y^{2}}{b^{2}}-\mathrm{z}=1$ & Elliptic hyperboloid & $\frac{x^{2}}{a^{2}}+\frac{y^{2}}{b^{2}}-\frac{z^{2}}{c^{2}}= \pm 1$ \\
paraboloid & $\frac{x^{2}}{a^{2}}+\frac{y^{2}}{b^{2}}=1$ & Cone & $\frac{x^{2}}{a^{2}}+\frac{y^{2}}{b^{2}}-\frac{z^{2}}{c^{2}}=0$ \\
Cylinder & $\frac{x^{2}}{a^{2}}-\frac{y^{2}}{b^{2}}=1$ & Parabolic cylinder & $x^{2}+2 a y=0$ \\
Hyperbolic cylinder & & & \\
\hline
\end{tabular}

\subsection{Creation and Matching of Feature Descriptors}

Each column of the matrix $\mathrm{T}$ in Section 2.2 represents the direction that the coordinate axis of the new coordinate system follows in the original coordinate system. In addition, the third column of $\mathrm{T}$ represents the axial direction of the quadratic surface in the original coordinate system. For any quadratic surface except the sphere, stable axial direction can always be obtained through data fitting. Hence, it can be used as a feature descriptor of the region. Note that the other two columns are incapable of producing stable direction for the perfect circular cylinder and circular cone, so they are not considered here.

In order to further improve the distinguishing ability of the feature descriptors, all points in the region are projected to the plane determined by their center of gravity and axial direction. The principal direction of the projected point clouds in the plane is obtained through PCA. The projection lengths of data along the two principal directions are incorporated into feature descriptors.

To sum up, the structure of the feature descriptor proposed in this paper is as follows:

Region Description D

\{

$\diamond$ Type of quadratic surface, $\boldsymbol{k}$;

$\diamond$ Axial direction, $v$;

$\diamond$ Middle point of the projected data along the axial direction, $\boldsymbol{o}$;

$\diamond$ Height of the projected data along the axial direction, $\boldsymbol{l 1}$; 


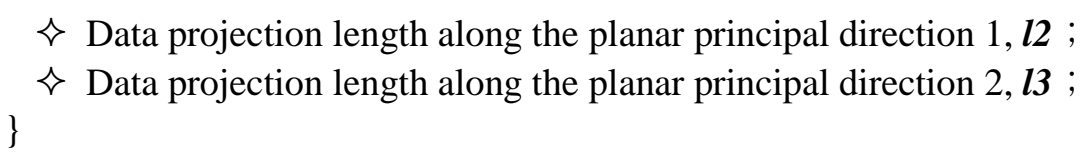

\subsection{Rough Registration of Point Clouds Based on Quadratic Features}

Obviously, if certain regions of two point clouds are matched, then the quadratic surface obtained through data fitting are of the same type. And the projection lengths of point clouds in these regions are similar along the axial direction to the lengths along the other two principal directions. So, a metric for measuring similarity between feature descriptors D1 and D2 is defined as follows:

$$
\zeta\left(D_{1}, D_{2}\right)= \begin{cases}0 & k_{1} \neq k_{2} \\ e^{-\sum_{i=1}^{3}\left|l_{1}-l i_{2}\right|} & \text { others }\end{cases}
$$

Steps for original matching of point clouds $\mathrm{P} 1$ and P2 based on (3) are given below:

Step1: for each quadratic surface from the point cloud P1, find regions from P2, which are most similar to the quadratic surface in P1 with the similarity above the threshold $\varepsilon$.

Step 2: if $\mathrm{N}(\mathrm{N} \geq 2)$ original matching regions are found out in Step1, then Choose two of the $\mathrm{N}$ original matching regions randomly. Based on the middle point $o$ and the axial direction $v$ of feature descriptors, compute the transformation between the two point clouds and the MSE error (i.e., $\mathrm{r}, \mathrm{t}$ and error e) using the method in [ppp].

Step 3: Transform P1 into P'1 via $r$ and t. Count the number of points (denoted by $\mathrm{Ne}$ ) in P'1 whose distance from P2 is below the threshold 2e.

Step 4: Repeat Steps 1-3. Record the transformation parameters which can minimize the error e/Ne. Define them as the final parameters $(\mathrm{R}$ and $\mathrm{T})$ for original transformation.

\subsection{ICP-Based Fine Registration}

After original registration, the two point clouds have overlapped to a large extent. Then, the ICP algorithm can be utilized to optimize results of original registration. Here, the point-to-plane rule is followed to search for the nearest point. And the distance threshold is defined as five times the optimal transformation error.

\section{Experiment and Analysis}

The proposed algorithm was implemented in $\mathrm{C}++$ on a PC platform with Intel Core i7$4770 \mathrm{CPU}$ and 3G Byte memory. During algorithm implementation, and the kd-tree was utilized to speed up the search for neighboring points.

As shown in Figure 2.a, the proposed algorithm was applied to the model with several cylinders and planes. In this model, a point cloud is clean, and the other cloud is corrupted with noise whose magnitude is equal to $10 \%$ of the average sampling interval. Figures 2.b, and 2.c, show the surface fluctuation calculation result obtained from the normal vector variation method, and the region segmentation result obtained from the proposed algorithm respectively. The intersection point threshold is defined as 1.5 times the average of surface fluctuation value, and the segmentation threshold is defined as 3 times the average sampling interval. From these figures, it can be seen that the proposed algorithm effectively represents fluctuation of surfaces and separates quadratic surfaces, producing correct segmentation results. Figure 2.d gives the point clouds registered by the feature description matching steps in Section 2.e. It can be seen that the proposed algorithm registers the two point clouds effectively at an average error of $0.15 \mathrm{~mm}$. Figure 2.f shows the fine registration result based on ICP. One can see that due to large original distance between the two models, ICP is stuck in local optimum, resulting in failure of model registration. 
a)

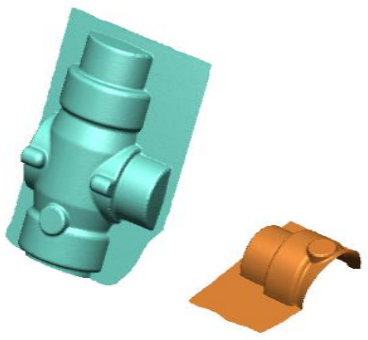

b)
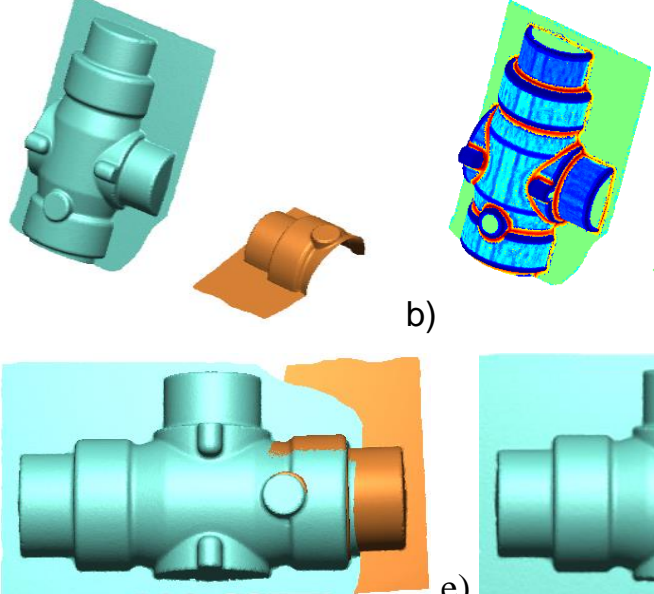
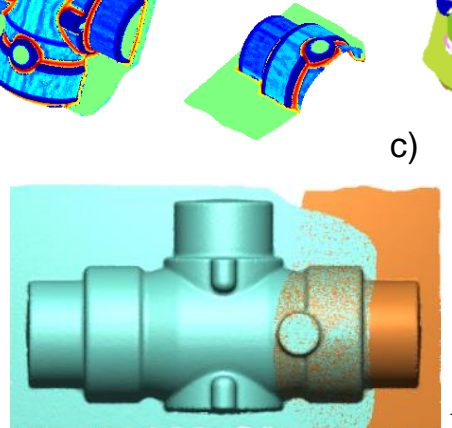

c)
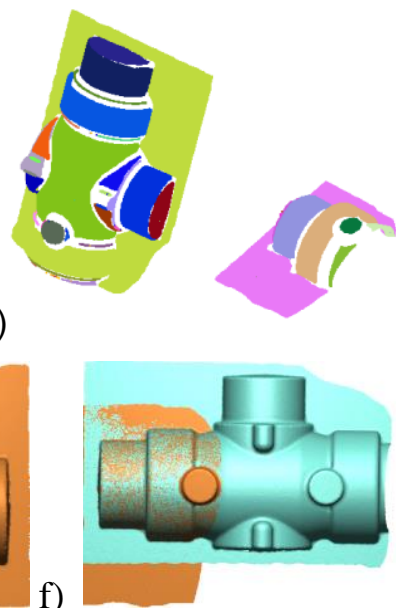

Figure 2. Data Registration of Point Clouds with Quadratic Surfaces

Our algorithm is robust to outliers which can be proved by the experiment in Figure 3 . Before experiment $20 \%$ outliers are added to both data. The outliers uniformly distributes in each data's bounding box and their distance to real data are at least 5\% of the diagonal distance. Because the normal of outliers are nearly random, so their surface fluctuation are much bigger than the normal points. That means when a threshold is used to remove points near the intersection of different regions from the point cloud, outliers had a high chance to be discard. Besides, the growing algorithm also won't group outliers into any normal point region because they have a much more distance to surface than the growing radius. Figure 3.b, is the result of outlier removing of our algorithm and the final align result is shown in Figure 3.c.

a)

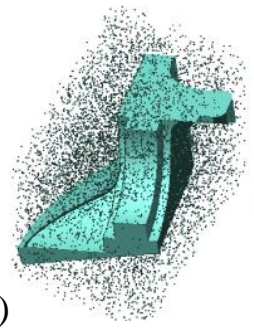

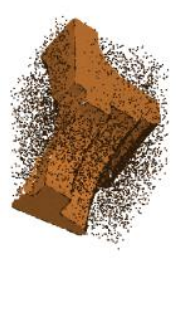

b)
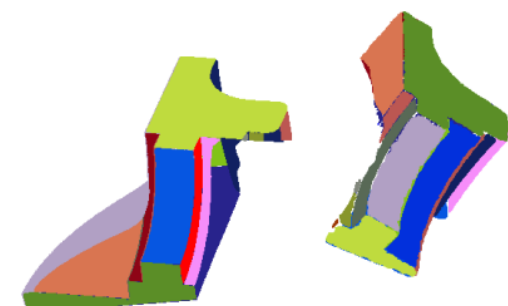

c)

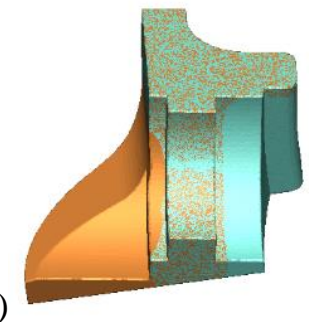

Figure 3. Data Registration of Point Clouds with Outliers

\section{Conclusion}

This paper proposes a novel algorithm for registering point clouds with quadratic surfaces. By deleting radically varying point cloud regions from the entirety of point clouds, the proposed algorithm can perform region segmentation correctly on point clouds, and recognizes the quadratic surfaces contained in the data. The proposed algorithm achieves rough registration of data by creating and matching feature descriptors. Fine registration is performed using ICP algorithm. Experimental results demonstrate insensitivity of the proposed algorithm to original stance of the model and 
outliers. Also, it has the ability to achieve higher success rates than the original ICP algorithm.

\section{Acknowledgements}

This work is sponsored in part by grants from Jiang- su Province Science Foundation for Youths (Grant No. BK20140892) and NUPTSF (Grant No. NY213038).

\section{References}

[1] D. Aiger, N. J. Mitra and D. Cohen-Or, "4-points congruent sets for robust pairwise surface registration", ACM Transactions on Graphics, vol. 27, no. 3, pp. 1-10

[2] I. Sipiran and B. Bustos, "3: A robust extension of the Harris operator for interest point detection on 3D meshes", The Visual Computer, vol. 27, no. 11, (2011), pp. 963-976.

[3] R. Gal and D. Cohen-Or, "Salient geometric features for partial shape matching and similarity", ACM Trans. Graph, vol. 25, no. 1, (2006), pp. 130-150.

[4] A. Zaharescu, E. Boyer and R. Horaud, "Keypoints and local descriptors of scalar functions on 2D manifolds", Int. J. Comput.Vis., vol. 100, no. 1, (2012), pp. 78-98.

[5] J. Stuckler and S. Behnke, "Interest point detection in depth images through scale-space surface analysis", in Proc. IEEE Int. Conf. Robot. Autom., (2011), pp. 3568-3574.

[6] F. Tombari, S. Salti and L. Di Stefano, "Unique shape context for 3D data description", in: ACM Workshop on 3D Object Retrieval, (2010), pp. 57-62.

[7] F. M. Sukno, J. L. Waddington and P. F. Whelan, "Rotationally invariant 3D shape contexts using asymmetry patterns", in: 8th International Conferenceon Computer Graphics Theory and Applications, (2013), pp. 1254-1262.

[8] Mian, M. Bennamoun, R. Owens, "On the repeatability and quality of keypoints for local feature-based 3D object retrieval from cluttered scenes”, Int. J. Comput. Vis., vol. 89, no. 2, (2010),pp. 348 -361.

[9] Y. Guo, S. Ferdous and B. Mohammed, "A novel local surface feature for 3D object recognition underclutter and occlusion”, Information Scicences, vol. 293, (2015), pp. 196-213

[10] M. A. FISCHLER and R. C. BOLLES, "Random sample consensus: A paradigm for model fitting with applications to image analysis and automated cartography", Commun. ACM, vol. 24, no. 6, pp. 381-395

[11] C. S. CHEN, Y. P. HUNG and J. B. CHENG, "RANSAC based DARCES: A new approach to fast automatic registration of partially overlapping range images", IEEE PAMI, vol. 21, no. 11 (1999) November, pp. 1229-1234

[12] N. GELFAND, N. J. MITRA, L. J. GUIBAS and H. POTTMANN, "Robust global registration", In Proc. SGP 3, (2005), pp. 197-206.

[13] Y. DIEZ, J. MARTA and J. SALVI, "Hierarchical normal space sampling to speed up point cloud coarse matching", Pattern Recognition Letters, vol. 33, no. 16, (2012), pp. 2127-2133.

[14] C. PAPAZOV and D. BURSCHKA, "Stochastic global optimization for robust point set registration", Comput. Vis. Image Underst., vol. 115, no. 12, (2011) Dec., pp. 1598-1609.

[15] D. AIGER, N. J. MITRA and D. COHEN-OR, "4-points congruent sets for robust surface registration", ACM TOG (SIG-GRAPH), vol. 27, no. 3, (2008), article no. 85, 1-10. 2, 3, 4.

[16] N. Mellado, D. Aiger and N. J. Mitra, "Super 4PCS:fast global pointcloud registration via smart indexing”, EUROGRAPHICS, vol. 33, no. 5, pp. 205-215

[17] P. J. BESL and N. D. MCKAY, "A method for registration of 3-d shapes", IEEE PAMI, vol. 14, no. 2, (1992) Feb., pp. 239-256.

[18] Y. CHEN and G. MEDIONI, "Object modelling by registration of multiple range images", Image Vision Comput., vol. 10, no. 3, (1992) April, pp. 145-155.

[19] L. SHANG and M. GREENSPAN, "Pose determination by potentialwell space embedding", In Proc. 3DIM 2, (2007), pp. 297-304.

[20] S. BOUAZIZ, A. TAGLIASACCHI and M. PAULY, "Sparse iterative closest point", CGF (SGP), vol. 32, no. 5, (2013), pp. 1-11.

[21] S. IZADI, D. KIM and O. HILLIGES, "Kinectfusion: Real-time 3d reconstruction and interaction using a moving depth camera", In Proc. UIST, 2, (2011), pp. 559-568.

[22] G. Frahm, M. Junker and A. Szimayer, "Elliptical copulas: applicability and limitations", Statistics \& Probability Letters, vol. 63, no. 3, (2003), pp. 275-286. 


\section{Author}

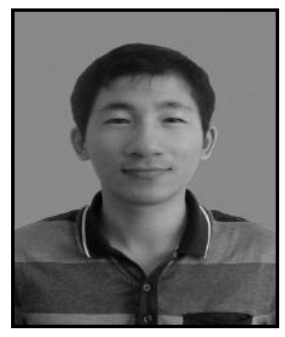

Nie Jianhui, received his M.S. degree in Control Theory and Control Engineering from Dalian Maritime University in Dalian, China. He is currently a lecturer in the College of Automation at Nanjing University of Posts and Telecommunications. His research interest is mainly in the area of Discrete Geometric Processing and Computer Vision Measurement. He has published several research papers in scholarly journals in the above research areas. 